\title{
High-resolution ultrasonography of cross-sectional area of median nerve compared with electro-diagnostic study in carpal-tunnel syndrome
}

\author{
Maryam Sahebari ${ }^{1 \#}$, Masoud Pezeshki Rad ${ }^{2 \#}$, Mohammad Ali Nahayati ${ }^{3}$, Morteza Saeidi ${ }^{4}$, Reza Boostani ${ }^{5}$, Yaser \\ Hamidian $^{6}$, Mahdieh Baghaei ${ }^{7^{*}}$ \\ ${ }^{1}$ Rheumatic Diseases Research Center, School of Medicine, Mashhad University of Medical Sciences, Mashhad, Iran \\ ${ }^{2}$ Surgical Oncology Research Center, Imam Reza Hospital, School of Medicine, Mashhad University of Medical Sciences, Mashhad, \\ Iran \\ ${ }^{3}$ Neurologist, Department of Neurology, Ghaem Hospital, School of Medicine, Mashhad University of Medical Sciences, Mashhad, \\ Iran \\ ${ }^{4}$ Department of Neurology, Ghaem Hospital, School of Medicine, Mashhad University of Medical Sciences, Mashhad, Iran \\ ${ }^{5}$ Department of Neurology, Ghaem Hospital, School of Medicine, Mashhad University of Medical Sciences, Mashhad, Iran \\ ${ }^{6}$ Departments of Radiology, Kashan University of Medical Sciences, Kashan, Iran \\ ${ }^{7}$ Department of Radiology, Imam Reza Hospital, Mashhad University of Medical Sciences, Mashhad, Iran
}

Carpal tunnel syndrome is a common chronic entrapment neuropathy. The present study evaluated the diagnostic ability of ultrasound (US) in measuring the cross-sectional area of the median nerve in the entrance of the carpal tunnel area in comparison with electrodiagnostic testing for diagnosing carpal tunnel syndrome. In this cross-sectional study, 253 patients with carpal tunnel syndrome based on American Academy of Neurology clinical symptoms were selected for participation. After screening, 200 patients were enrolled in the study. Wrist US and electrodiagnostic findings for patients were compared. Data were analyzed by SPSS. There was a good agreement between sonographic and electrodiagnostic findings [right hand $(\mathrm{kappa}=0.71)$, left hand $(\mathrm{kappa}=0.78)$ ]. For the right and left hands, respectively, ultrasound sensitivity $(89 \%$ vs $90 \%)$, specificity ( $90 \%$ vs $89.2 \%$ ), positive predictive value ( $84.7 \%$ vs $94.7 \%$ ), and negative predictive value $(75.7 \%$ vs $81.6 \%$ ) were calculated. High-resolution ultrasound of the median nerve cross-sectional area is an accurate test for diagnosing carpal tunnel with an acceptable level of diagnostic ability. In comparison with the electrodiagnostic test, ultrasonography can provide more practical information about anatomical changes in the tunnel, which can be considered in selecting treatment options.

Keywords: carpal tunnel syndrome, electromyography, entrapment neuropathy, ultrasonography.

\section{Introduction}

Carpal tunnel syndrome (CTS) is a chronic peripheral neuropathy which is particularly present in middle-aged women [1]. The risk for CTS has been estimated about $10 \%$, and its incidence rose from 258 to 367 per 100,000 between the years 1981 and 2005 [2].

Median nerve compression within the carpal tunnel by transverse carpal ligament (TCL) in the wrist leads to CTS. The exact etiology of carpal tunnel syndrome is still unknown. In aggregate, local and systemic etiologies are involved in its pathogenesis. The most important local factors are bony fractures, blunt trauma, ganglion, lipoma, and repeated wrist movements (mostly career-related ones). The most common systemic etiologies are diabetes, thyroid dysfunction, pregnancy, renal failure, and nerve enlargement secondary to myeloma. Mean carpal tunnel pressure is about $14.3 \mathrm{mmHg}$ in normal individuals, but it increases to $43 \mathrm{mmHg}$ in patients with carpal tunnel syndrome [3, 4].

CTS manifestations include pain and paresthesia in the median nerve pathway, such as numbness in the fingers, which deteriorates during sleep time or repetitive hand movements [1].

Disease manifestations and etiology are influenced by gender. CTS occurs bilaterally in $60 \%$ of patients. Female sex, obesity, and older age are some of the most important risk factors for idiopathic CTS. Diabetes and hypothyroidism are two other major risk factors for it. Trauma is frequent in men with CTS. Pregnancy and \# These authors equally contributed to this study.

* Corresponding Author: Mahdieh Baghaei, Email: baghaeim891@mums.ac.ir, Tel: +98 5138022534

Received: 11 December 2016; Accepted: 30 April 2017 
rheumatoid arthritis are associated with it as well $[5,6]$.

Usually, clinical features and provocative clinical examinations such as Phalen's test are enough to make a diagnosis. However, in patients with mild or suspected nonspecific symptoms, diagnostic studies are required [7].

Currently, the nerve conduction study (NCS) is the most reliable test for confirming CTS. Median nerve distal motor latency is considered the most specific neurological test for diagnosing CTS, but its sensitivity is moderate. Recent studies have proposed that peripheral nerve ultrasonography (US) could be a complementary diagnostic tool for CTS [8].

The present study evaluated the diagnostic ability of US at the cross-sectional area of the median nerve in comparison with the electrodiagnostic method employed in diagnosing carpal tunnel syndrome.

\section{Materials and Methods}

\section{Patient selection:}

The study population included patients with pain and numbness in the median nerve innervations area, especially at night, and/or positive Phalen's or Tinel's tests. American Academy of Neurology clinical symptoms and patient history were considered as inclusion criteria [8]. To avoid interfering parameters, patients with diabetes mellitus, chronic CTS with previous wrist surgery or median nerve release, corticosteroid intra-canal injection, hypothyroidism, rheumatoid arthritis, recent hand or wrist trauma, clinical manifestations of polyneuropathy, median nerve anomalies such as bifid median nerve diagnosed by US, and pregnant women were excluded from the study.

Clinical examinations for CTS and screening for the mentioned exclusion criteria were performed by a single rheumatologist.

This study was approved by the Ethical Committee of Mashhad University of Medical Sciences. All patients signed an informed consent form.

\section{Laboratory tests}

Thyroid function and fasting blood sugar were assessed in all patients, and other paraclinical examinations were conducted when appropriate according to clinical data.

\section{Electrodiagnostic tests}

Electrodiagnostic tests, the gold standard for diagnosing CTS [9], were carried out according to the recommended guidelines of the American Association of Electrodiagnostic Medicine (AAEM) [10, 11]. The Nicolet Viking Select System (EMG matrix light EP, Micromed Inc, Italy) was used for NCS. All patients underwent electrodiagnostic tests in the same room with equivalent temperature and humidity conditions. Nerve conduction velocity (NCV) tests were conducted to measure distal latency, amplitude, and distance of median nerve. By EMG, distal motor nerve latency (DML) and distal peak latency were calculated. The neurologist and radiologist who performed the diagnostic tests were blind to the results of the physical examinations.

According to Stevens criteria, CTS severity was designated as below:

Normal: normal NCV or negative electrodiagnostic findings for CTS

Mild: prolonged mean distal peak latency more than $3.5 \mathrm{~m} / \mathrm{s}$ and/or sensory nerve action potential (SNAP) amplitude lower than normal

Moderate: sensory defects like mild CTS with prolonged distal peak latency more than $4.4 \mathrm{~m} / \mathrm{s}$

Severe: prolonged mean distal motor latency and distal peak latency with the absence of SNAP and CMAP, absence or low voltage action potential, spontaneous muscle activity in EMG [7].

\section{Ultrasonographic assessment}

Within three days after the electrodiagnostic tests, all patients were evaluated by high-resolution sonography with a $10 \mathrm{mhz}$ linear array transducer (Esaote Mylab, Italy). A single independent radiologist performed all US examinations. All patients underwent US assessment in the same room, in a comfortable position, with their forearms placed on a table. Patient palm position was supine. Distal wrist crease was considered as the US starting point (Fig. 1). A general overview of the median nerve was obtained in the first step. The transducer (with gel) was laid directly upon the patient's wrist skin. The median nerve circumference was calculated using the continuous boundary trace method. The severity of CTS was calculated by the median nerve circumference in sonography:

Normal: $\leq 10 \mathrm{~mm}^{2}$.

Mild: $10 \mathrm{~mm}^{2}<$ median nerve circumference $\leq$ $13 \mathrm{~mm}^{2}$.

Moderate: $13 \mathrm{~mm}^{2}<$ median nerve circumference $\leq 15 \mathrm{~mm}^{2}$.

Severe: more than $15 \mathrm{~mm}^{2}$.

\section{Statistical analysis}

Data was analyzed by the Statistical Package for Social Science (SPSS Inc, version 16.0 for Windows). Data was represented as mean $\pm \mathrm{SD}$. Normality of data was assessed using the Kolomogrove-Smirnov test. Other parametric and non-parametric tests were used to 
analyze data. The MacNemar test was used to compare sonographic and electrodiagnostic results for patients. The Kapa test evaluated the agreement between the two above-mentioned methods. The Spearman correlation test was used to measure correlations. A p value less than 0.05 was considered significant.

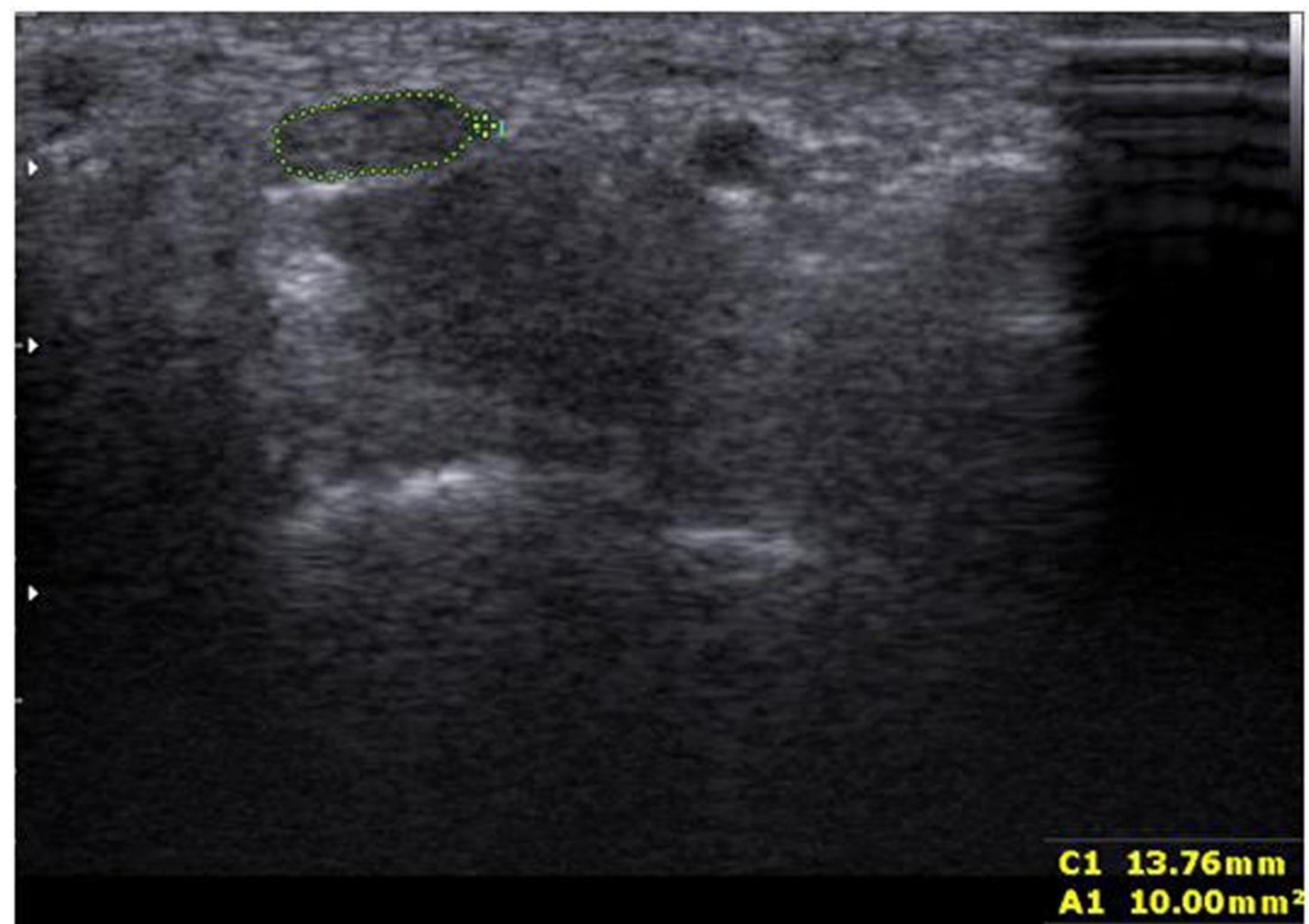

Fig. 1. Ultrasonic cross-sectional measurement of median nerve in wrist

\section{Results}

Initially, 253 patients with a clinical diagnosis of CTS entered the study. Out of 253 patients, 48 were excluded by special screening before NCV due to occult diabetes, hypothyroidism, or other exclusion criteria. The demographics and characteristics of the study population are presented in Table 1.

There was a good agreement between the sonographic and electrodiagnostic findings (Table 2). Ultrasound sensitivity ( $89 \%$ vs $90 \%)$, specificity $(84.7 \%$ vs $89.2 \%$ ), positive predictive value (93.5\% vs $94.7 \%$ ), and negative predictive value $(75.7 \%$ vs $81.6 \%)$ were calculated for the right versus the left hand, respectively. The correlation between US and the electrodiagnostic test for the right hand were $P<0.001$, $\mathrm{r}_{\mathrm{s}}=0.79$ and for the left hand were $P=0.001, \mathrm{rs}=0.77$ ).

Table 2 shows the agreement between ultrasound and electrodiagnostic tests in carpal tunnel syndrome.

\section{Discussion}

The present study evaluated the correlation between ultrasonography findings and electrodiagnostic test results, the gold standard for diagnosing CTS in patients who were suspected for carpal tunnel syndrome. The results confirmed the good agreement between those two diagnostic tests. There was a statistically significant agreement between sonographic and electrodiagnostic findings in both hands in the current study.

Some studies have shown that swelling of the median nerve in all segments and flexor retinaculum bowing had a specificity of about $60 \%[12,13]$.

In the current study, the ultrasound diagnostic ability was relatively acceptable.

The current study demonstrated that false negative CTS in electrodiagnostic examination was $9.4 \%$ for left and $11 \%$ for right hand. These percentages were higher in previous studies such as Chen et al. and van Doesburg et al. [14-17]. This difference might be due to the use of different instruments or methods. 
Table 1. Characteristics of carpal tunnel syndrome patients

\begin{tabular}{ll}
\hline Characteristics & Number(\%) \\
\hline Gender & $30(14.6)$ \\
Male & $175(85.4)$ \\
Female & \\
Age group (y) & $60(29.3)$ \\
$<40$ & $123(60)$ \\
$40-60$ & $22(10.7)$ \\
$>60$ & \\
Hand Involvement & $33(16.1)$ \\
Left & $60(29.3)$ \\
Right & $112(54.6)$ \\
Bilateral & \\
US findings & $71(34.6)$ \\
Normal & $70(34.1)$ \\
Mild CTS & $41(20)$ \\
Moderate CTS & $23(11.2)$ \\
Sever CTS & \\
Electrodiagnostic findings & $65(31.7)$ \\
Normal & $55(26.8)$ \\
Mild CTS & $51(24.8)$ \\
Moderate CTS & $22(10.7)$ \\
Sever CTS &
\end{tabular}

Table 2. Agreement between ultrasound and electrodiagnostic test in Carpal tunnel syndrome

\begin{tabular}{|c|c|c|c|c|}
\hline $\begin{array}{c}\text { Diagnostic } \\
\text { procedure/ } \\
\text { hand }\end{array}$ & $\begin{array}{c}\text { Positive } \\
\text { report by } \\
\text { two } \\
\text { techniques } \\
N(\%)\end{array}$ & $\begin{array}{l}\text { Negative } \\
\text { report by } \\
\text { two } \\
\text { techniques } \\
N(\%)\end{array}$ & $\begin{array}{c}\text { Positive } \\
\text { by US } \\
\text { and } \\
\text { Negative } \\
\text { by NCV } \\
\mathrm{N}(\%)\end{array}$ & $\begin{array}{c}\text { Positive } \\
\text { by NCV } \\
\text { and } \\
\text { Negative } \\
\text { by US N } \\
(\%)\end{array}$ \\
\hline Right hand & $130(82.4 \%)$ & $50(24.39 \%)$ & $9(4.3 \%)$ & $16(7.8 \%)$ \\
\hline \multicolumn{5}{|c|}{ kappa:0.71 (agreement) } \\
\hline Left hand & $126(61.46)$ & $58(28.29)$ & $7(3.41)$ & $13(6.345)$ \\
\hline \multicolumn{5}{|c|}{ kappa:0.78 (agreement) } \\
\hline
\end{tabular}

Although electrodiagnostic tests are accurate and the gold standard for diagnosing carpal tunnel syndrome, $10 \%$ to $25 \%$ of cases would be missed using this test, depending on disease severity or method of performance [17]. Yesildag et al. demonstrated that a median nerve circumference of more than $10.5 \mathrm{~mm}$ is sensitive (89\%) and specific $(94.7 \%)$ for CTS [6].

Moran confirmed that, although US assessment of the median nerve cross-sectional area at the carpal tunnel inlet could be considered a good alternative compared with electrodiagnostic studies for CTS. However, sonography did not determine the severity of CTS. In Moran's study, a cross-sectional area greater than $13 \mathrm{~mm}$ was diagnostic for CTS [18]. The current study confirmed those results.

In 1992, it was proposed that high resolution realtime ultrasound was the same as CT scan and MR findings in carpal tunnel syndrome [19].
One of the highest diagnostic ability levels for US (more than 96\%) was calculated in a study by Miedany [20]. That study suggested that ultrasound assessment and CT scan should be performed for all patients who had a positive history and a clinical examination. Moreover, electrodiagnostic tests should only be considered for patients with normal sonography and CT scan results [20]. In the current study, US accuracy was acceptable, but its specificity was lower than that in the Miedany report; such result might be related to differences in radiologist experience or methods of patient recruitment. For example, the researchers in Miedany's study used patient-oriented data (the Arabic version of the Boston Carpal Tunnel Questionnaire (ABCTQ)) which might reduce the number of patients without CTS and increase the accuracy of diagnostic tests [21]. The ultrasonography method used in the current research was the same as that of Miedany's study.

The current findings were in agreement with those of previous studies. Although the accuracy of US was the same as that of the electrodiagnostic test, ultrasound is preferable because of its easy performance and painless procedure.

Some points of strength of the current study were the acceptable number of patients, the site (in the carpal tunnel) of sonographic examination, choosing idiopathic CTS patients, and excluding cases of secondary nerve hypertrophy due to metabolic disorders.

This study was not without limitations. For example, having US conducted by one radiologist may lead to the over- or under-diagnosis of CTS.

\section{Conclusion}

Results of the current study confirmed that using highresolution ultrasound imaging to study the median nerve in its entrapment neuropathy is an accurate test for diagnosing carpal tunnel with an acceptable level of diagnostic ability. Moreover, when compared with electrodiagnostic test, US provides more practical information about anatomical changes in the carpal tunnel, which is important for treatment options.

\section{Conflicts of interest}

The authors declare no conflicts of interest.

\section{Acknowledgments}

This article was extracted from the thesis prepared by Dr. M.A Nahayati to fulfill the requirements needed for earning a specialty degree in Neurology. The Research Council of Mashhad University of Medical Sciences, 
Mashhad, Iran, is appreciated for financially supporting this study grant number [88093]. The authors are also grateful to all patients for their kind participation.

\section{References}

1. Bradley WG, Daroff RB Fenichel GM, Jankovic J. Neurology in clinical practice. $6^{\text {th }}$ ed. Philadelphia: Elsevier; 2012; 1926-1930

2. Bongers FJ, Schellevis FG, van den Bosch WJ, van der Zee J. Carpal tunnel syndrome in general practice (1987 and 2001): incidence and the role of occupational and nonoccupational factors. $\boldsymbol{B r} \boldsymbol{J}$ Gen Pract 2007; 57: 36-39

3. Duncan I, Sullivan P, Lomas F. Sonography in the diagnosis of carpal tunnel syndrome. AJR $\boldsymbol{A m}$ J Roentgenol 1999; 173(3): 681684. doi: 10.2214/ajr.173.3. 10470903.

4. Lee $\mathrm{CH}$, Kim TK, Yoon ES, Dhong ES. Correlation of highresolution ultrasonographic findings with the clinical symptoms and electrodiagnostic data in carpal tunnel syndrome. Ann Plast Surg 2005; 54(1): 20 23. doi: 10.1097/01.sap.0000141 942.27182.55.

5. Ablove RH, Moy OJ, Peimer CA, Wheeler DR, Diao E. Pressure changes in Guyon's canal after carpal tunnel release. $J$ Hand Surg Br 1996; 21(5): 664-665. doi: 10.1016/s0266-7681(96)8015 5-0.

6. Yesildag A, Kutluhan S, Sengul $\mathrm{N}$, Koyuncuoglu HR, Oyar O, Guler K, et al. The role of ultrasonographic measurements of the median nerve in the diagnosis of carpal tunnel syndrome. Clin Radiol 2004; 59(10): 910-5. doi: 10.1016/j.card.2004.03.020.

7. Chiotis K, Dimisianos N, Rigopoulou A, Chrysanthopoulou A, Chroni E. Role of anthropometric characteristics in idiopathic carpal tunnel syndrome. Arch Phys Med Rehabil 2013; 94(4): 737-44. doi 10.1016/j.apmr.2012.11.017.

8. Baert AL, Knauth M, Sartor K. High-Resolution sonography of the peripheral nervous system. $2^{\text {nd }}$ ed. Berlin-Verlag Heidelberg:
Springer, 2008; 84-93.

9. Visser LH1, Smidt MH, Lee ML High-resolution sonography versus EMG in the diagnosis of carpal tunnel syndrome. J Neurol Neurosurg Psychiatry 2008; 79(1): 63-7. doi: 10.1136/jnnp. 2007.115337.

10. American Academy of Neurology. Practice parameter for carpal tunnel syndrome (summary statement): report of the Quality Standards Subcommittee of the American Academy of Neurology. Neurology 1993; 43(11): 2406-9. doi: 10.1212/wnl. 43.11.2406.

11. Miyamoto H, Halpern EJ, Kastlunger M, Gabl M, Arora R, Bellmann-Weiler R.Carpal tunnel syndrome: diagnosis by means of median nerve elasticity-improved diagnostic accuracy of US with sonoelastography. Radiology 2014; 270(2): 481-6. doi: 10.1148/radiol.13122901.

12. American Association of Electrodiagnostic Medicine, American Academy of Neurology, and American Academy of Physical Medicine and Rehabilitation. Practice parameter for electrodiagnostic studies in carpal tunnel syndrome: summary statement. Muscle Nerve 2002; 25(6): 918-22. doi: $10.1002 /$ mus. 10185 .

13. Miwa $\mathrm{T}, \mathrm{Miwa} \mathrm{H}$ Ultrasonography of carpal tunnel syndrome: clinical significance and limitations in elderly patients. Intern Med 2011; 50(19): 215761. doi: 10.2169/ internalmedicine. 50.5771.

14. Sarría L, Cabada T, Cozcolluela R, Martínez-Berganza T, García S. Carpal tunnel syndrome: usefulness of sonography. Eur Radiol 2000; 10(12): 1920-5. doi: 10.1007/s003300000502.

15. Chen SF, Lu CH, Huang CR, Chuang YC, Tsai NW, Chang $\mathrm{CC}$, et al. Ultrasonographic median nerve cross-section areas measured by 8-point "inching test" for idiopathic carpal tunnel syndrome: a correlation of nerve conduction study severity and duration of clinical symptoms. BMC Med Imaging 2011; 11(1): 22. doi: 10.1186/1471-2342-1122.

16. Witt JC, Hentz JG, Stevens JC. Carpal tunnel syndrome with normal nerve conduction studies. Muscle Nerve 2004; 29(4): 515522. doi: 10.1002/mus.20019.

17. van Doesburg MH, Henderson J, Yoshii Y, Mink van der Molen $\mathrm{AB}$, Cha SS, An KN. Median nerve deformation in differential finger motions: ultrasonographic comparison of carpal tunnel syndrome patients and healthy controls. J Orthop Res 2012; 30(4): 643-8. doi: 10.1002/jor. 21562.

18. Moran L, Perez M, Esteban A, Bellon J, Arranz B, del Cerro M. Sonographic measurement of cross-sectional area of the median nerve in the diagnosis of carpal tunnel syndrome: correlation with nerve conduction studies. J Clin Ultrasound 2009; 37(3): 125-31. doi: 10.1002/jcu.20551.

19. Buchberger W, Judmaier W, Birbamer G, Lener M, Schmidauer C. Carpal tunnel syndrome: diagnosis with highresolution sonography. AJR Am J Roentgenol 1992; 159(4): 793-8. doi: 10.2214/ajr.159.4.1529845.

20. El Miedany YM, Aty SA, Ashour S. Ultrasonography versus nerve conduction study in patients with carpal tunnel syndrome: substantive or complementary tests? Rheum 2004; 43(7): 88795. doi: 10.1093/rheumatology/ keh190.

21. Levine DW, Simmons PB, Koris MJ, Daltroy LH, Hohl GG, Fossel AH. A self-administered questionnaire for assessment of severity of symptoms and functional status in carpal tunnel syndrome. J Bone Joint Surg Am 1993; 75(11): 1585-92. doi: 10.2016/00004623-19931100000002 . 